\title{
Article \\ A Leakage Model of Contact Mechanical Seals Based on the Fractal Theory of Porous Medium
}

\author{
Xingya Ni, Chenbo Ma *, Jianjun Sun, Yuyan Zhang ${ }^{(D)}$ and Qiuping Yu \\ College of Mechanical and Electronic Engineering, Nanjing Forestry University, Nanjing 210037, China; \\ nixy@njfu.edu.cn (X.N.); sunjianjun@njfu.edu.cn (J.S.); yuyan_zhang@njfu.edu.cn (Y.Z.); \\ yuqiuping03@njfu.edu.cn (Q.Y.) \\ * Correspondence: machenbo@njfu.edu.cn
}

Citation: Ni, X.; Ma, C.; Sun, J.; Zhang, Y.; Yu, Q. A Leakage Model of Contact Mechanical Seals Based on the Fractal Theory of Porous Medium. Coatings 2021, 11, 20. https://dx.doi.org/10.3390/coatings 11010020

Received: 12 November 2020 Accepted: 24 December 2020 Published: 27 December 2020

Publisher's Note: MDPI stays neutral with regard to jurisdictional claims in published maps and institutional affiliations.

Copyright: () 2020 by the authors. Licensee MDPI, Basel, Switzerland. This article is an open access article distributed under the terms and conditions of the Creative Commons Attribution (CC BY) license (https: / / creativecommons.org/ licenses/by/4.0/).

\begin{abstract}
A theoretical model for calculating the leakage rate of contact mechanical seals based on the fractal theory of the porous media, which can consider the real seal contact interface and objectively reflect the flow of the interfacial fluid from a microscopic perspective, is established. In order to obtain the microstructural parameters of the porous media included in the leakage model, such as the fractal dimension and the maximum pore diameter, the real seal contact interface obtained from experiments is reconstructed, a contact model between the dynamic and static rings is proposed, and then the calculation methods for the interface characteristic parameters are provided. Numerical simulation results show that as the contact pressure increases from 0.05 to $0.5 \mathrm{MPa}$, the interface porosity and the maximum pore diameter decreases gradually. Furthermore, the fractal dimension of the pore area increases and the leakage rate of the interface decreases from 0.48 to $0.33 \mathrm{~mL} / \mathrm{h}$. The proposed method provides a novel way of calculating the leakage rate of contact mechanical seals.
\end{abstract}

Keywords: contact mechanical seal; leakage model; porous media; fractal theory

\section{Introduction}

The leakage of contact mechanical seals is a common problem in industrial applications. More specifically, the leakage failure is often out of expectation for new equipment or a newly installed mechanical seal. Failure of the mechanical seal often leads to serious losses and may even result in catastrophic combustion, explosion, harmful medium leakage and accidents. Therefore, it is of significant importance to investigate the performance of the mechanical seal and reduce the end face leakage, improve the predictability and reliability of the leakage, and make a reasonable maintenance period.

Previous literature [1] indicates that mechanical seals are widely used as an important part of the rotary equipment to reduce leakage, save energy and protect the environment, so that they have become an important research field in sealing technology. Mechanical seals include two basic types: the contact type and the noncontact type. Contact mechanical seals have a simple structure in comparison with the noncontact mechanical seals. Moreover, contact mechanical seals do not require complex end-face liquid or the gas supply for the blockage control system. Furthermore, they have low manufacturing and maintenance costs and can achieve reasonable sealing performance and reliability at the design condition. Therefore, contact mechanical seals have always been the research hotspot for scholars and engineers worldwide [2-5].

In order to effectively control and reduce the leakage of contact mechanical seals, the main leakage passage between the end faces of the dynamic and static rings should be investigated [6]. Then, the corresponding evolutionary theory and external factors should be applied to establish a scientific and reasonable leakage calculation model. For this reason, scholars have carried out many studies in this regard. Heinze [7] found that when the mechanical seal leaks, the fluid flow in the small clearance obeys the law of hydrodynamics and the fluid flow has the laminar flow pattern. He established the 
leakage model for the plane with ideally smooth clearance. Mayer [1] simplified the leakage passage of a micro passage with an equal radial height and a uniform gap width, where the gap width depends on the roughness of the sealing surface. Lubeck [8] studied various factors affecting the performance of the mechanical seal ring, including the flexural deformation, force deformation, thermal deformation, processing methods and material properties. He applied the Fourier transformation to study the leakage with the waviness interface. Subsequently, Lebeck [9] and Elhanafi et al. [10] investigated the roughness and the waviness of the sealing contact surface and put forward a model for the leakage passage gap with the taper. Considering the variations of the taper angle of the seal face caused by the wear, Green [11] studied the change rule of the time-dependent flow in the simplified leakage passage clearance. Sun et al. [12] used fractal parameters to characterize the scale irregularity of the roughness, waviness and taper of the sealing surface. They deduced the time-dependent fractal model of the leakage passage on the sealing interface. Francesc Pérez-Ràfols et al. [13] established a model composed of state-of-the-art models for the contact mechanics between rough metal surfaces and for the liquid flow through the rough aperture in-between them. Li et al. [14] built a model based on fractal theory; the contact between surfaces of dynamic and static rings is simplified to the contact between the rough surface and the ideal rigid plane. The leakage model, wear model, and thermal deformation model of the contact mechanicals seal are established, and the fractal leakage model considering the wear and thermal deformation is established by taking them all into consideration. The essence of the foregoing leakage channel model is to simplify the pore between rough contact interfaces into the parallel or cosine-shaped interstices along the radial equal height of the interface. This conversion remarkably simplifies the calculation process. However, micro-convex bodies and pits on the two surfaces of the seal generate strong disorders and random characteristics. When these surfaces contact each other, the leakage channels between sealing interfaces are formed. When only simplified geometric models are employed to describe the leakage rate, channels that do not connect the contact surfaces to penetrate the sealing surface are neglected. Therefore, the calculated leakage rate based on these models is normally higher than the measured value [15].

For this reason, many leakage channel models have been proposed so far to calculate the leakage rate and clarify the leakage theory. Bao et al. [16] considered a correlation between the fluid flow and the seepage between end faces and established a seepage channel model between end faces of contact mechanical seals. In order to obtain the porosity and the maximum pore radius, the leakage rate between the end faces can be calculated by the Darcy equation. It provides a new idea for the study of the leakage channel of the sealing interface. However, the Darcy equation is an empirical expression, which can only cover the macroscopic characteristics of the flowing fluids and cannot describe the characteristics of fluids precisely at the pore scale. Moreover, there are many differences between the hypothetical and real sealing interfaces in the simulation research, which adversely affect the accuracy of the calculation results. According to the two-dimensional percolation and the contact mechanics theory, Bottiglione et al. [17] established a two-dimensional model for the percolation leakage channel for the static sealing interface. They studied the influence of the surface fractal morphology and the applied load on the critical size of the leakage channel. Inspired by this investigation, Yu et al. [18] studied the permeability characteristics of the interface of contact mechanical seals and the variation of the critical time of the permeability passage height. Zhou et al. [19] declared that the two-dimensional percolation channel model does not consider the three-dimensional morphology of the actual rough surface. Therefore, a percolation model for three-dimensional contact between dynamic and static rings of contact mechanical seals was constructed, and a method for determining percolation of pore masses was proposed. Sun et al. [20,21] proposed the hypothesis of the single-layer mesh percolation at the sealing interface. They compared the percolation threshold of the sealing interface with different mesh layers. Moreover, they compared the porosity of the sealing interface at contact pressures of the end face. Then they established the leakage channel model as a function of the porosity of the sealing interface body. The 
establishment of the leakage passage model based on the percolation theory makes it possible to study the fluid flow in the disordered interface of the mechanical seal. However, this model is still in the preliminary stage. The calculation of the leakage rate is mainly focused on the leakage at a single percolation point, while the actual leakage passage may have multiple percolation points and different bifurcation forms so that calculated results were usually smaller than the measured value [21].

Recently, the fractal correlation theory of the porous media has attracted the attention of scholars because it can effectively deal with the random disordered flow in complex objects. Patel et al. [22] created a model that could be used to understand the relative magnitude of challenges in sealing gases/liquids at true viscosities. Various types of artificially generated surfaces were considered in the model, and a parametric study was conducted. However, the effect of tortuosity was ignored in their calculation of leakage rate, and the influence of interface contact was calculated by the theory of contact mechanics. Zhang et al. [23] studied a model that was aiming at static sealing, the calculation of leakage rate is realized by combining the fractal model of porous media and the contact model, and a very clear idea is given. The calculation of the maximum pore diameter in the model is determined by the method of equivalent hydraulic diameter. The characteristics of this paper are that, for the contact mechanical seal, the contact deformation is obtained by collecting the actual surface morphology and combining it with numerical simulation. Based on the analysis of the image after the surface contact deformation, a new calculation method of the maximum pore diameter is proposed, and then the leakage rate is calculated based on the Hagen-Poiseuille equation using the fractal model of porous media. It is intended to apply the fractal theory of the porous media to establish a novel calculation model for the leakage passage. It is expected that the present study can provide a new way of calculating and predicting the leakage rate of contact mechanical seals and effectively revealing the leakage theory.

\section{Model}

\subsection{Leakage Rate Calculation Model Based on the Fractal Theory of the Porous Media}

The porous media refers to the solid media containing a large number of irregular pores or voids. Generally, the porous media has the following characteristics [24]:

- It is solid with a large number of pores;

- Pores are interconnected in varying degrees;

- Pores have different sizes and shapes;

- The fluid can flow in the internally connected network of pores.

The transport of the liquid, gas, electricity, heat, sound and the wave in the porous media is called the porous media transport. The liquid flow on the sealing face is an example of porous media transport. In fact, the liquid transport characteristics and transport mechanism directly affect the final sealing performance. Reviewing the literature [1] shows that the investigation methods for the transport characteristics and mechanism of the porous media are usually divided into three categories: numerical simulation, experimental measurement and analytical analysis. It should be indicated that the analytical analysis method is widely used to analyze the correlation between macroscopic transport characteristics and the microscopic mechanism of porous media. In fact, the analytical method is quite effective for porous media with simple and regular geometries, including the square, equilateral triangle and cylindrical structures. However, the size, shape and spatial distribution of the pore and particles in most practical porous media are random. In other words, the conventional analytical method cannot be applied in practical applications. With the development of research, it has been found that the pore size distribution or the pore and solid microstructures in the porous media are fractal structures or can be approximated with fractal structures. Therefore, the fractal theory has been applied to study the correlation between the macroscopic transport properties and the microscopic mechanisms of the porous media. 
In fractal theory, the measurement parameter of fractal objects $M(L)$ and the measurement scale $L$ satisfies the following scale correlation [25]:

$$
M(L) \propto L^{D_{f}}
$$

where $D_{f}$ denotes the fractal dimension and $M(L)$ can be the density or the mass of the object, the surface area of the object or the length of the curve.

Furthermore, the distribution number of pores $N$ and the pore size in the porous media is expressed in the form below [26,27]:

$$
N(L \geq \lambda)=\left(\frac{\lambda_{\max }}{\lambda}\right)^{D_{f}}
$$

Equation (2) indicates that the total number of pores $N_{t}$ can be written as:

$$
N_{t}\left(L \geq \lambda_{\min }\right)=\left(\frac{\lambda_{\max }}{\lambda_{\min }}\right)^{D_{f}}
$$

where $\lambda, \lambda_{\max }$ and $\lambda_{\min }$ are the normal, maximum and minimum pore diameters, respectively.

Applying the differential operator on Equation (2) results in the following formula:

$$
-\mathrm{d} N=D_{f} \lambda_{\max }^{D_{f}} \lambda^{-\left(D_{f}+1\right)} \mathrm{d} \lambda
$$

Equation (4) shows the total number of voids in the interval between $\lambda$ and $\lambda+\mathrm{d} \lambda$. Dividing Equation (4) into Equation (3) yields the following equation:

$$
-\frac{\mathrm{d} N}{N_{t}}=D_{f} \lambda_{\min }^{D_{f}} \lambda^{-\left(D_{f}+1\right)} \mathrm{d} \lambda=f(\lambda) \mathrm{d} \lambda
$$

where $f(\lambda)=D_{f} \lambda_{\min }^{D_{f}} \lambda^{-\left(D_{f}+1\right)}$ is the probability density of the pore distribution in the porous media and satisfies the following requirements:

$$
\int_{-\infty}^{\infty} f(\lambda) \mathrm{d} \lambda=\int_{\lambda_{\min }}^{\lambda_{\max }} f(\lambda) \mathrm{d} \lambda=1
$$

Equation (6) is established on the condition that [28]:

$$
\left(\frac{\lambda_{\min }}{\lambda_{\max }}\right)^{D_{f}}=0
$$

If Equation (7) is not satisfied, the fractal theory cannot be used to study the porous media. In most porous media in the practical application, including sealed interfaces, $\lambda_{\min } / \lambda_{\max }<10^{-2}$. In other words, these media satisfy Equation (7). Therefore, it is feasible to apply fractal theory and method to study the transport characteristics of the porous media.

For a typical porous medium, as shown in Figure 1, the flow rate $q(\lambda)$ of the fluid satisfies the modified Haagen-Poiseulle equation [29,30], with the following assumptions that the fluid does not deform porous medium (fluid pressure $<<$ contact pressure), and the surface effects-surface tension, adsorption and drag are neglected. Furthermore, the fluid is 100\% saturation and single fluid. In addition, it is isothermal flow and incompressible Newtonian fluid:

$$
q(\lambda)=\frac{\pi}{128} \frac{\Delta P}{L_{t}(\lambda)} \frac{\lambda^{4}}{\mu}
$$

where $\Delta P$ is the pressure of the sealing medium. $\mu$ is the hydrodynamic viscosity. Moreover, $L_{0}$ denotes the representative length of the channel, and it is expressed as $L_{0}=$ 
$\left[\frac{1-\phi}{\phi} \frac{\pi D_{f} \lambda_{\max }^{2}}{4\left(2-D_{f}\right)}\right]$ [31]. Finally, $L_{t}(\lambda)$ is the length of the pore channel with the size of land, and it is defined as $L_{t}(\lambda)=\left(L_{0} / \lambda\right)^{D_{T}}$, where $D_{T}$ is the fractal dimension of the tortuosity, as shown in Figure 1, which is analytically expressed in the form below [31]:

$$
D_{T}=1+\frac{\ln \left\{\left[\tau_{a v}\left(D_{f}+D_{T}-1\right) / D_{f}\right]\right\}}{\ln \left(L_{0} / \lambda_{\min }\right)}
$$

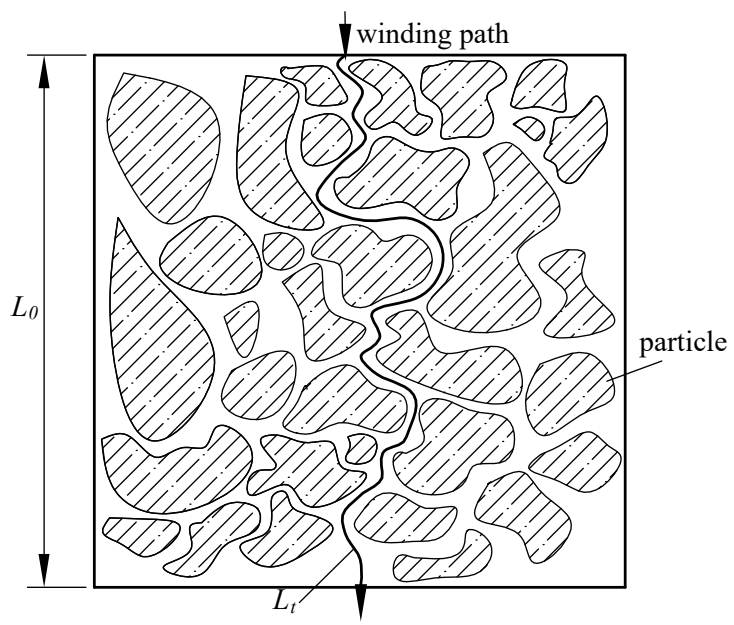

Figure 1. The winding paths in the porous medium.

In the foregoing equation, $\tau_{a v}$ is the average tortuosity, which can be calculated as the following [31]:

$$
\tau_{a v}=\frac{1}{2}\left[1+\frac{1}{2} \sqrt{1-\phi}+\sqrt{1-\phi} \sqrt{(1 / \sqrt{1-\phi}-1)^{2}+\frac{1}{4}} / 1-\sqrt{1-\phi}\right]
$$

Considering Equations (4) and (8), the total fluid flow through a unit with a crosssection $Q$ is obtained as:

$$
\begin{aligned}
& Q=-\int_{\lambda_{\min }}^{\lambda_{\max }} q\left({ }_{\lambda}\right)_{\mathrm{d}} N\left({ }_{\lambda}\right)=\frac{\pi}{128} \frac{\Delta P}{\mu} \frac{A}{L_{0}} \frac{L_{0}^{1-D_{r}}}{A} \frac{D_{f}}{3+D_{T}-D_{f}} \lambda_{\max }^{3+D_{T}} \times\left[1-\left(\frac{\lambda_{\min }}{\lambda_{\max }}\right)^{3+D_{T}-D_{f}}\right]= \\
& \frac{\pi}{128} \frac{\Delta P}{\mu} \frac{A}{L_{0}} \frac{L_{0}^{1-D_{r}}}{A} \frac{D_{f}}{3+D_{T}-D_{f}} \lambda_{\max }^{3+D_{T}} \times\left[1-\left(\frac{\lambda_{\min }}{\lambda_{\max }}\right)^{D_{f}}\left(\frac{\lambda_{\min }}{\lambda_{\max }}\right)^{3+D_{T}-D_{f}}\right]
\end{aligned}
$$

In the two-dimensional space, $1<D_{f}<2$ and $1<D_{T}<2$. Applying these constraints in equation (11) proves that $3+D_{T}-2 D_{f}>0$, in another word, $0<\left(\frac{\lambda_{\min }}{\lambda_{\max }}\right)^{3+D_{T}-D_{f}}<1$. Based on the judgment Equation (7), Equation (11) can be transformed into the following form:

$$
Q=-\int_{\lambda_{\min }}^{\lambda_{\max }} q(\lambda) \mathrm{d} N(\lambda)=\frac{\pi}{128} \frac{\Delta P}{\mu} \frac{A}{L_{0}} \frac{L_{0}^{1-D_{r}}}{A} \frac{D_{f}}{3+D_{T}-D_{f}} \lambda_{\max }^{3+D_{T}}
$$

Equation (12) does not contain any empirical constants, and the six parameters included in it all have definite physical interpretations. Among these parameters, the hydrodynamic viscosity $\mu$ is a known parameter, and the differential pressure driving force $\Delta P$ can be obtained in accordance with the actual working conditions. Therefore, in order to calculate the flow rate of the porous media, only the microstructural parameters of the porous media, including the porosity $\phi$, maximum pore diameter $\lambda_{\max }$ and the fractal dimension $D_{f}$, should be obtained. 


\subsection{Acquisition of Microstructural Parameters of the Porous Media at the Interface of the Real Mechanical Seals}

Although the surface of the dynamic and static rings of the contact mechanical seals seems smooth, actually, there are a huge number of micro-convex bodies and pits of different sizes and shapes on the seal surface. During the startup and shutdown operations, some micro-convex bodies between the two surfaces have direct contact, while other parts are partially connected so that pores and leakage channels are formed. As a result, leakage may even occur in the normal operation because the contact mechanical seals are in the mixed lubrication state. Unlike the study on common porous media, the surfaces of dynamic and static rings of mechanical seals have relative motion so that the mechanical seal surface is accompanied by a certain degree of friction and wear. However, the corresponding interface is in a solid phase, and the interface pores are connected to each other in different degrees. This indicates that although the porous medium theoretically varies along time, the sealing interface of the contact mechanical seals meets the basic characteristics of the porous media [32].

As a preliminary study, this paper only takes the seal face after running-in as the research object, assuming that its surface morphology does not change over a long period of time. Considering the surface morphology and random characteristics of the contact, the dynamic sealing interface can be simplified to a static porous media with a statistical geometry model so that the porous media theory can be employed to carry out the research [33,34].

Figure 2a presents an illustration of the contact mechanical seal, which is composed of one pair of end faces perpendicular to the rotating axis, under the action of fluid pressure and the contact force. In the present analysis, the material of the static ring of the contact mechanical seal is graphite. The inner and outer diameters at the end face of the static ring are 52 and $60 \mathrm{~mm}$, respectively. For the sake of computational reduction, a local region of the contact surface, as shown in Figure 2b, is analyzed in the present model by assuming that the surface profile exhibits periodic characteristics.

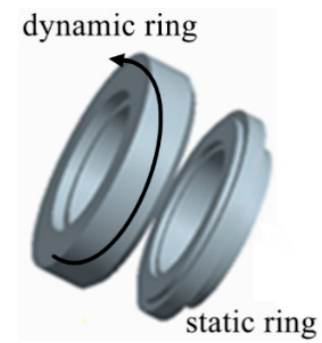

(a)

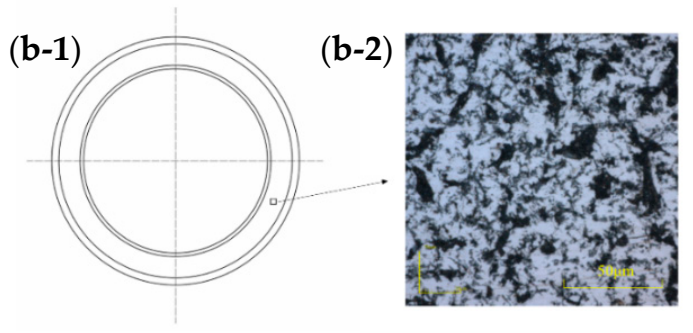

(b)

Figure 2. Illustrations of the tested seal rings and the local topography on the surface of the static ring: (a) 3D model of tested seal rings; (b) where the present model get; (b-1) 2D model of static ring; (b-2) local topography on the surface.

In the actual operation process, we selected the inner side, the middle position and the outer side of the seal ring to collect and process. It is found that the leakage rates of different regions have little difference, as shown in Table 1. Therefore, this paper selects the area in the middle position to carry out the simulation. Furthermore, as the surface topography exhibits random and periodic characteristics, the dimension selected for the model also has little influence on the leakage rate. 
Table 1. Leakage rates calculated in different locations.

\begin{tabular}{|c|c|c|c|}
\hline \multirow{2}{*}{$\begin{array}{c}\text { Contact Pressure } \\
(\mathrm{MPa})\end{array}$} & \multicolumn{3}{|c|}{ Leakage Rate $\left(\mathrm{mL} \cdot \mathrm{h}^{-1}\right)$} \\
\hline & $\begin{array}{l}\text { Region in the Inner } \\
\text { Side }\end{array}$ & $\begin{array}{l}\text { Region in the } \\
\text { Middle Position }\end{array}$ & $\begin{array}{c}\text { Region in the Outer } \\
\text { Side }\end{array}$ \\
\hline 0.1 & 0.453 & 0.451 & 0.452 \\
\hline 0.2 & 0.406 & 0.408 & 0.403 \\
\hline 0.3 & 0.382 & 0.385 & 0.385 \\
\hline 0.4 & 0.351 & 0.352 & 0.351 \\
\hline 0.5 & 0.314 & 0.313 & 0.315 \\
\hline
\end{tabular}

As a preliminary study of the model, some assumptions are made. In the contact analysis, only the elastic-plastic contact is considered, and the bearing capacity of the fluid between the interfaces is not considered. Assuming that the surface morphology of the seal ring is randomly distributed, the part selected can represent the leakage rate of the sealing ring surface. The influence of wear on the surface morphology during the relative movement of seal rings is not considered.

For the sake of clarity, the whole flow chart for the calculation of the flow rate of the porous media in mechanical contact seal is demonstrated in Figure 3. The details about the reconstruction of the sealing face, contact analysis and the determination of microstructural parameters of the porous media will be described in the following sections.

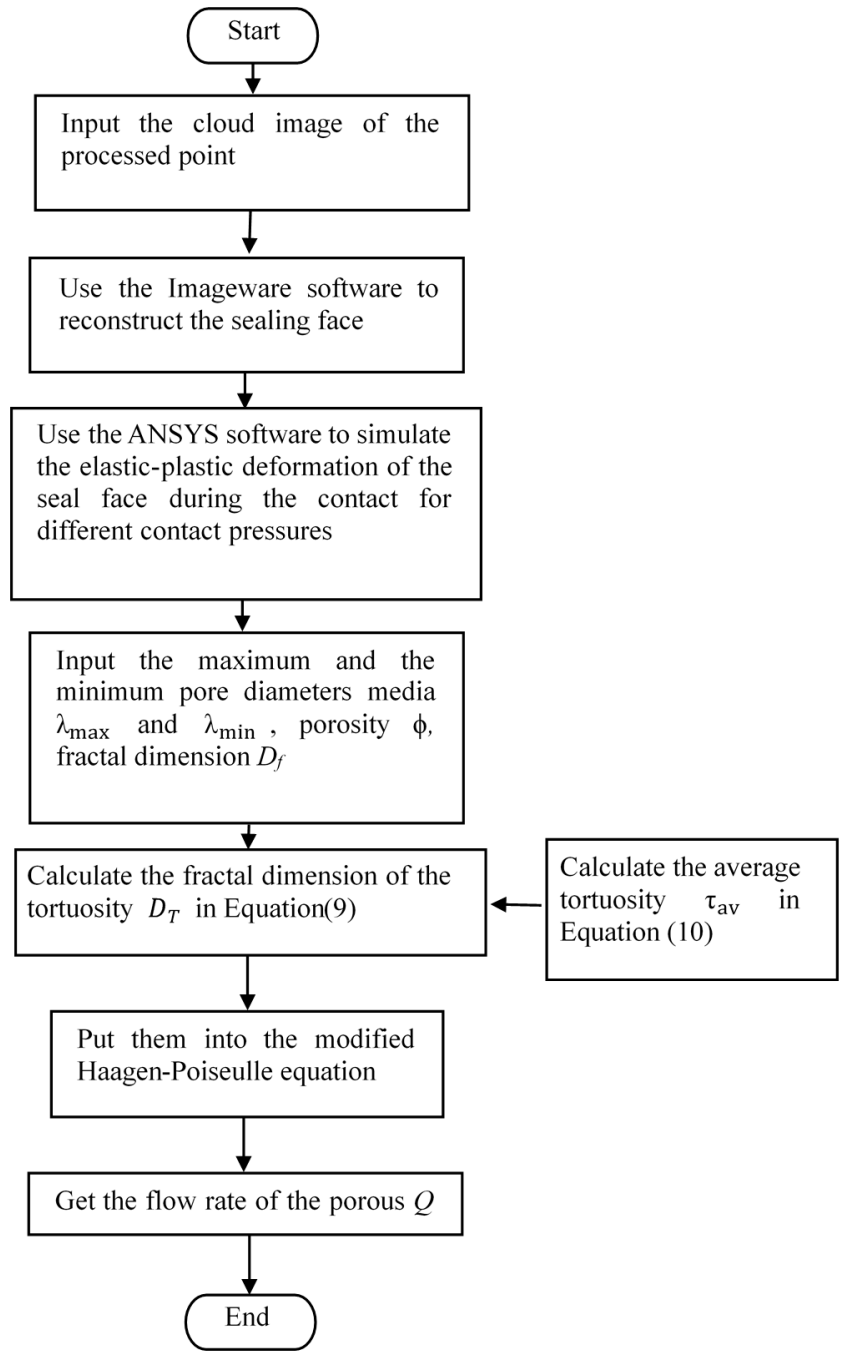

Figure 3. Flowchart for the calculation of the flow rate of the porous media. 
2.2.1. Establishment of the Geometric Model and Contact Model for the Porous Medium at the Interface of Mechanical Seals

In order to obtain the microstructural parameters of the mechanical seal interface, including the porosity $\phi$, maximum pore diameter $\lambda_{\max }$, fractal dimension $D_{f}$, the corresponding variation law and the leakage rate, it is necessary to establish a geometric model for the porous medium based on the real mechanical seal interface.

Based on the principle of reverse engineering, laser confocal microscopy is used to collect the real seal face morphology and convert it into the point cloud data. Figure $2 b$ shows the surface morphology of the stationary ring, which is measured at the horizontal resolution of $0.12 \mu \mathrm{m}$ and the $Z$-axis resolution of $0.01 \mu \mathrm{m}$, with the measurement range of $128 \mu \mathrm{m} \times 128 \mu \mathrm{m}$. Figure 4 shows the point of clouds after processing. Through performing the noise reduction, smoothing and sparse processing of the point cloud data, the Imageware software (version 13.2) is employed to reconstruct the sealing face and accomplish the reverse engineering. The reconstructed seal face morphology, which has the same dimension as that in Figure 2b, is presented in Figure 5.

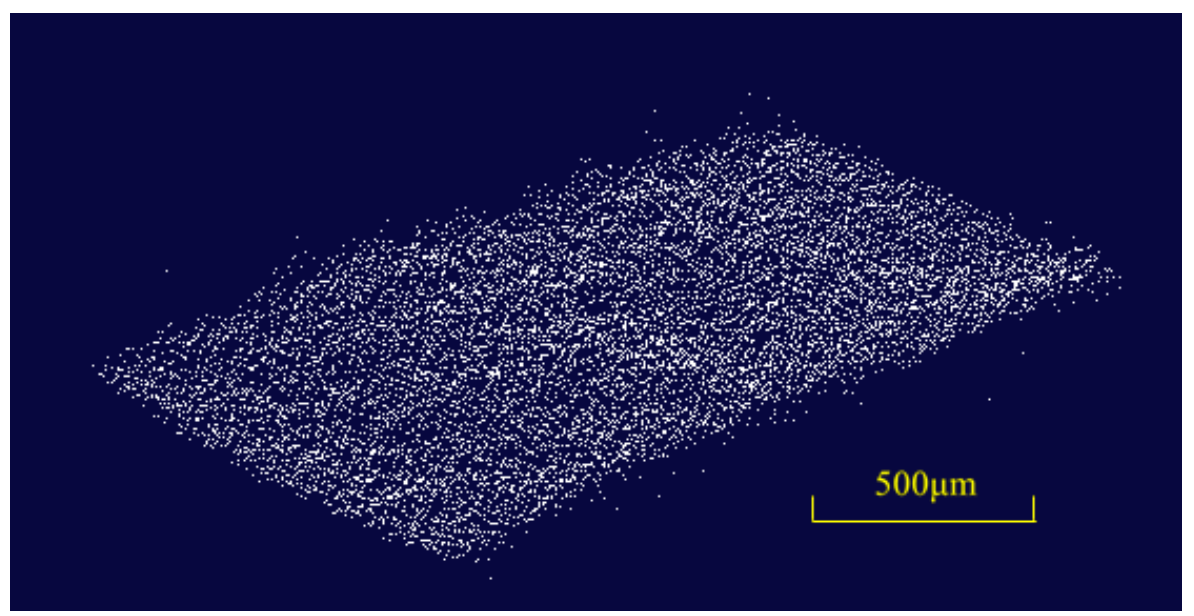

Figure 4. Point clouds after processing.

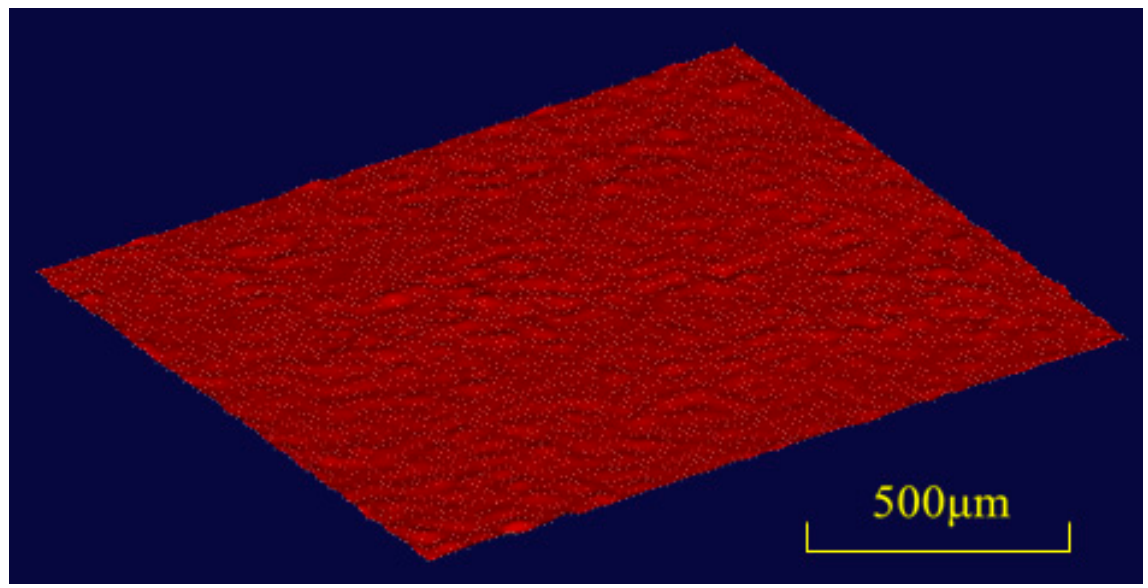

Figure 5. Reconstructed seal end surface morphology.

According to the reconstructed seal face morphology, three-dimensional modeling software is applied to perform the solid modeling of the seal face. Since the dynamic and static rings of contact, mechanical seals are usually made of soft-hard collocation, the elastic modulus of the material of the dynamic ring is much higher than that of the static ring. Moreover, the roughness of the end surface of the dynamic ring is smaller than that of the static ring. Therefore, the complex contact between the end faces of mechanical 
seals can be simplified as the contact between a rigid, smooth plane and an elastic-plastic rough plane and then the contact model of the dynamic and static rings can be constructed. It should be indicated that the ANSYS software (version 18.0) is applied to simulate the elastic-plastic deformation of the seal face during the contact for different contact pressures. The elastic modulus and Poisson's ratio of the static ring are set as $E=2 \times 10^{10} \mathrm{~Pa}$ and $v=0.29$, respectively. A kinematic hardening constitutive model is used to describe the plastic behavior of the static ring with the yield strength $\sigma=4.5 \times 10^{8} \mathrm{~Pa}$. The static ring is modeled by 894,382 numbers of high-quality hexahedral elements. Fixed support is used to limit all degrees of freedom of the lower surface of the static ring, and the radial displacement of the dynamic ring is limited. Uniform pressure is applied to the upper surface of the dynamic ring to simulate the contact pressure on the end face in the actual working condition. In this model, the contact pressure increases from 0.02 to $0.52 \mathrm{MPa}$, with an increment of $0.02 \mathrm{MPa}$. In addition, the contact type is set to frictional contact, the friction coefficient is set to 0.15 , the contact surface is the rough upper surface of the static ring, and the target surface is the lower surface of the dynamic ring. All simulations are performed in ANSYS Workbench (ANSYS, Inc., Pittsburgh, PA, USA) using the static structural analysis.

Figure 6 shows the contact model between the dynamic and static rings. Through numerical simulations in ANSYS Workbench, the deformation of the contact surface and then the morphology of the deformed static ring, as shown in Figure 7 can be obtained. Based on the deformed morphology of the static ring, the geometric model of the porous media at the mechanical seal interface can be constructed, and its microstructural parameters can be calculated.

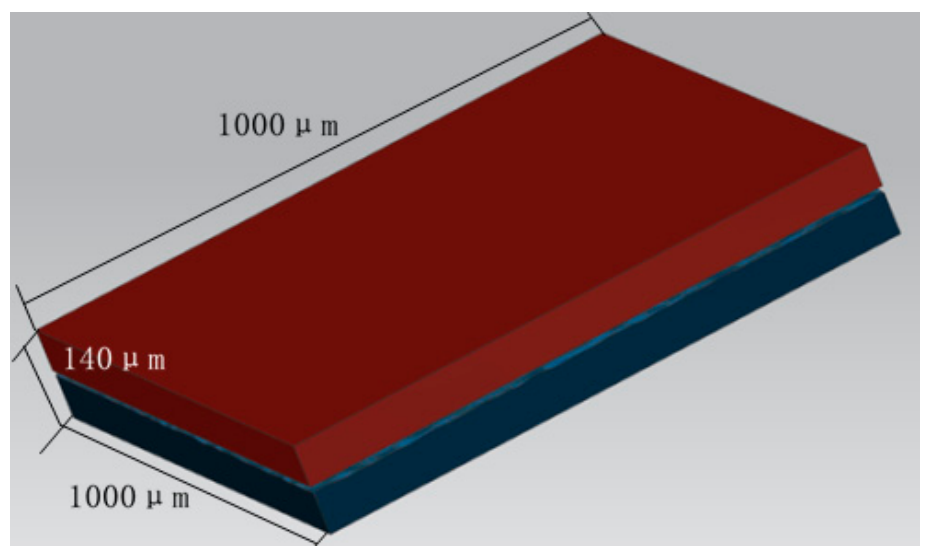

Figure 6. The contact model of seal rings.

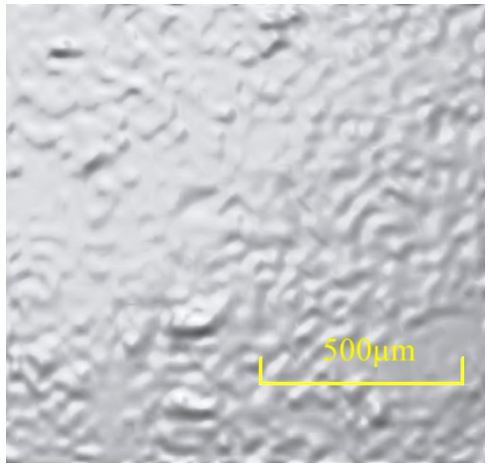

(a)

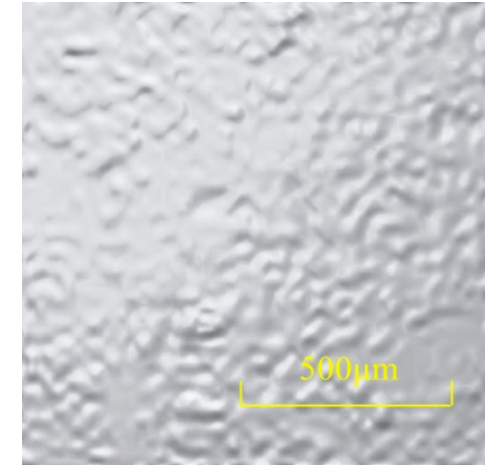

(b)

Figure 7. The surface topography under different contact pressures: (a) $0.02 \mathrm{MPa}$; (b) $0.16 \mathrm{MPa}$. 


\subsubsection{Calculating the Porosity $\phi$ and Its Variation at Different Contact Pressures}

Porosity $\phi$ is an important parameter for describing the pore distribution in the porous media, which is defined as:

$$
\phi=\frac{V_{p}}{V_{s}+V_{p}}
$$

where $V_{p}$ and $V_{\mathrm{S}}$ denote the pore and the solid skeleton volumes, respectively.

Figure 8 shows the schematic diagram of the sealing interface. It indicates that in order to calculate the porosity, only volumes of the skeleton and the pore in the enclosed space, consisting of the highest and lowest peaks and valleys of the surface, can be calculated. It should be indicated that positions of the highest and lowest peaks can be determined by the point cloud data of the surface.

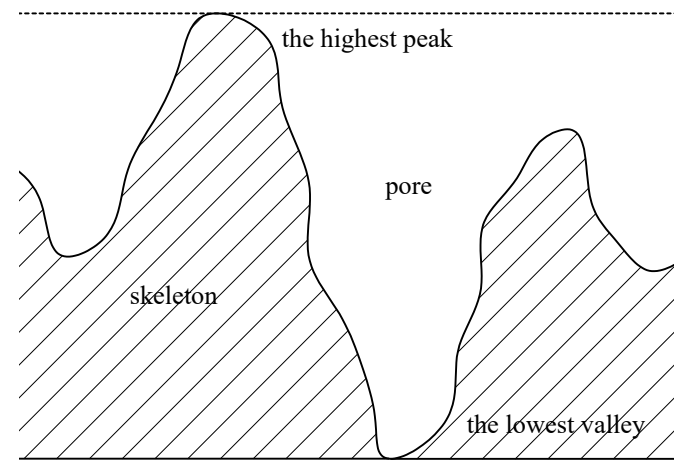

Figure 8. Shape diagram of the surface on the static ring.

Figure 9 shows the variation of the porosity of the sealing interface at different contact pressures. It is observed that the porosity decreases gradually as the contact pressure increases. This is more pronounced in the early stage of the loading. It can be interpreted as the following: during the early stage of the loading, because of the small radian of the top of the micro-convex body involved in the contact model and the small number of the micro-convex body, the micro-convex body is rapidly crushed under the action of a smaller external load, which makes the sealing clearance rapidly smaller, so the porosity is also rapidly reduced. Moreover, as the contact pressure increases, more micro-convex bodies are involved in the contact. At a constant extrusion of external loads, the solid is flattened, and voids are gradually filled, which further reduces the porosity. However, the rate of reduction gradually decreases. It is observed that when the contact pressure exceeds $0.35 \mathrm{MPa}$, the porosity approaches $45 \%$.

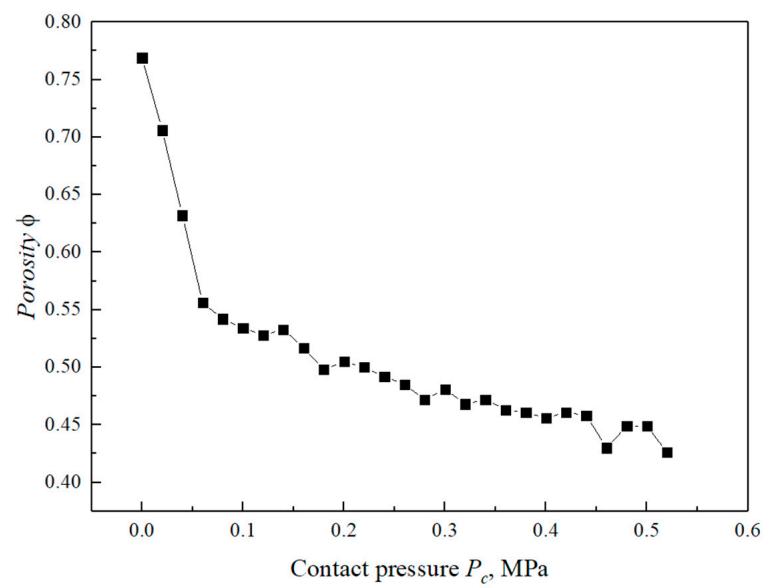

Figure 9. The influence of the contact pressure on the porosity. 


\subsubsection{Calculating Fractal Dimension of the Pore Area and Its Variation at Different Contact Pressures}

Fractal dimension $D_{f}$ is an important parameter to characterize the fine structure of the surface profile. In the present study, the box-counting method is applied to calculate the fractal dimension of the two-dimensional pore area of the seal face at different contact pressures. According to the principle of box-counting, the calculation steps are as follows:

- Preprocessing the image of the end face of the static ring (see Figure 7), including the gray transformation, smoothing filtering and noise reduction, and choose an appropriate threshold to binarize the image;

- Read the binary image and convert it into the numerical matrix $H$;

- Cover the numerical matrix with squares of different sizes, and count the number of squares containing pore in different sizes, as the total number of squares is called $N_{r}$;

- Then, the least-squares method should be applied to fit the straight line for the distribution of $\ln r$ against $\ln N_{r}$. It should be indicated that the absolute value of the slope of the fitted line is the box-counting dimension.

Figure 10 shows the variation of the fractal dimension $D_{f}$ of the pore area at the end of the static ring at different pressures. It is observed that the fractal dimension of the pore area increases gradually as the contact pressure increases. This variation can be interpreted as follows: Fractal dimension is a parameter describing the complexity of the fractal structure. In fact, the larger the fractal dimension, the finer the structure. It is found that as the contact pressure increases, the rough peaks of the seal face are flattened continuously, voids of the porous media are more filled by the solid, and the end surface becomes flat gradually. Therefore, the end surface microstructure becomes finer, short-wavelength components increase, and the fractal dimension increases gradually.

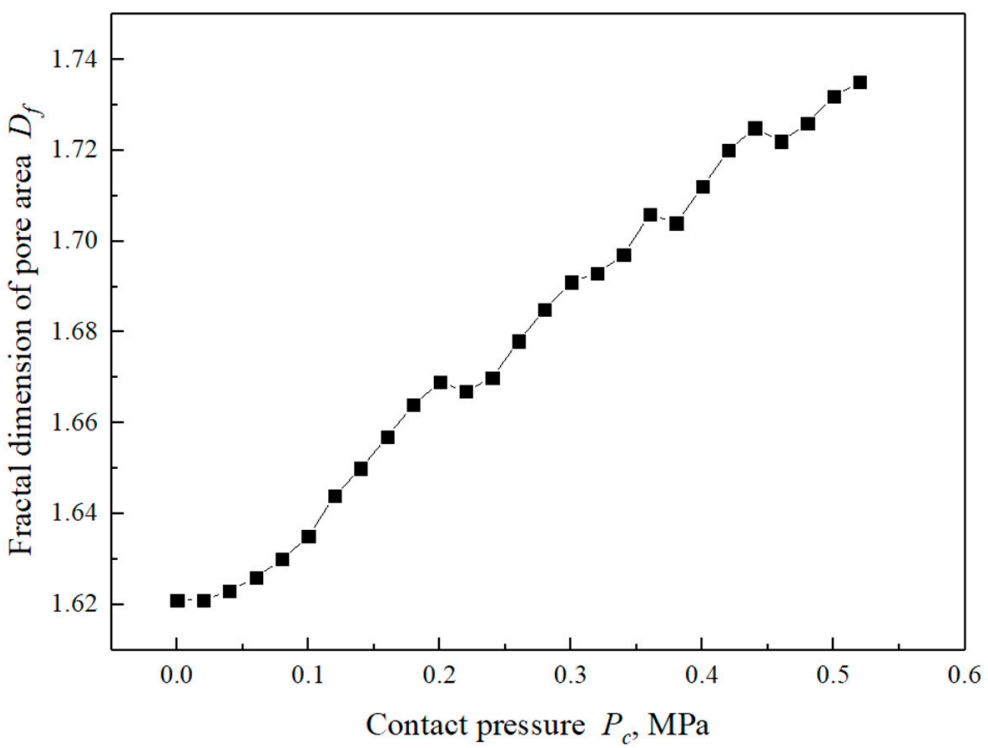

Figure 10. The influence of the contact pressure on the fractal dimension of pore area.

\subsubsection{Calculating the Maximum Pore Diameter and Its Variation at Different} Contact Pressures

Generally, it is a challenge to directly measure the maximum pore diameter $\lambda_{\max }$ of the porous media through the experiment. Although the high-resolution Scanning Electron Microscope (SEM) micrograph of the porous media can reflect the size and distribution of the pore to a certain extent, this method alone cannot be applied to obtain the ideal structure data of the maximum pore in a narrow space. In order to calculate the maximum pore diameter of the porous media for the sealing interface (i.e., $\lambda_{\max }$ ), the leakage passage connecting many irregular pores is considered as a tubular passage with different diameters. Then a method for calculating the maximum pore diameter is proposed in the present 
study, which is based on the box-counting principle. The corresponding concrete idea is as follows: When calculating the maximum pore diameter of the porous media, the numerical matrix representing the seal interface morphology is covered by boxes with different side lengths $r$, and the number of boxes containing target voxels (voids) for different box side lengths is recorded. Table 2 shows the obtained results as an example to calculate the maximum pore diameter.

Table 2. Number of boxes covered with holes for different edge lengths of boxes.

\begin{tabular}{cc}
\hline Side Length $\boldsymbol{r}$ & Number of Box $\boldsymbol{N}_{\boldsymbol{r}}$ \\
\hline 512 & 1 \\
256 & 4 \\
128 & 16 \\
64 & 64 \\
32 & 256 \\
16 & 982 \\
8 & 3714 \\
4 & 11,394 \\
2 & 29,396 \\
1 & 78,256 \\
\hline
\end{tabular}

Furthermore, Figure 11a illustrates a schematic diagram of the maximum pore area. It indicates that in order to find the maximum diameter of the pore, it is necessary to determine the edge length of the box when it covers the maximum pore area in the process of continuously decreasing the edge length of the box. Figure $11 \mathrm{~b}$ shows that the actual edge length corresponding to the edge length of the box is the maximum diameter of the pore. Moreover, it is observed that the box shape for the target coverage is a regular square, while the pore shape is random and irregular. Therefore, when the box is required to cover the pore area completely, the pore part cannot exactly fill the box. In other words, the box size corresponding to the edge length is larger than the actual maximum pore diameter.

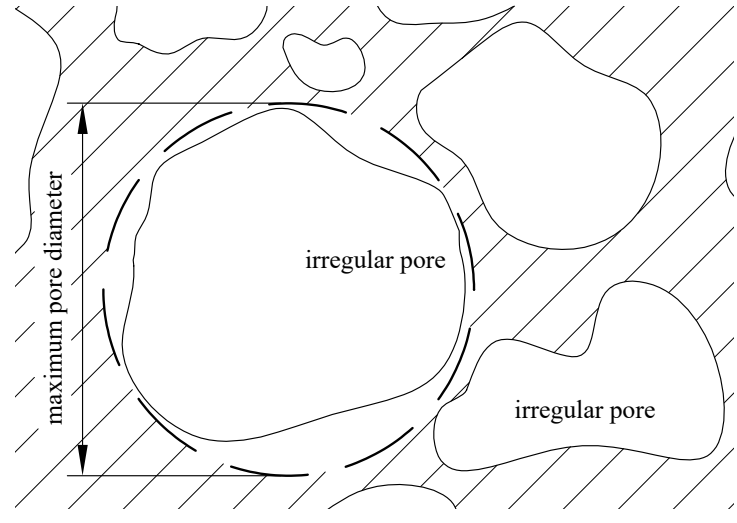

(a)

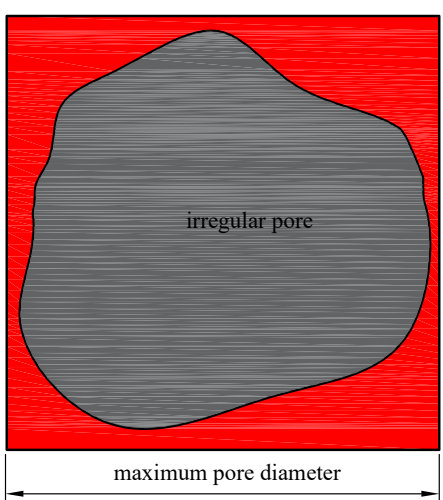

(b)

Figure 11. Schematic diagram of the maximum pore and the box cover: (a) the maximum pore area; (b) the actual edge length.

In order to determine the corresponding edge length of the box when it can cover the pore area completely, the correlation between the different edge lengths of the box and the number of boxes containing the pore part is studied in accordance with the data in Table 2. Figure 12 illustrates the obtained distribution between the above-mentioned parameters. It is found that the number of boxes containing voids increases exponentially as the size of boxes decreases. Then the data corresponding to the beginning and end stages of the curve is fitted by straight lines, where it is found that the corresponding box edge length at the intersection of two lines is about 4.1. 


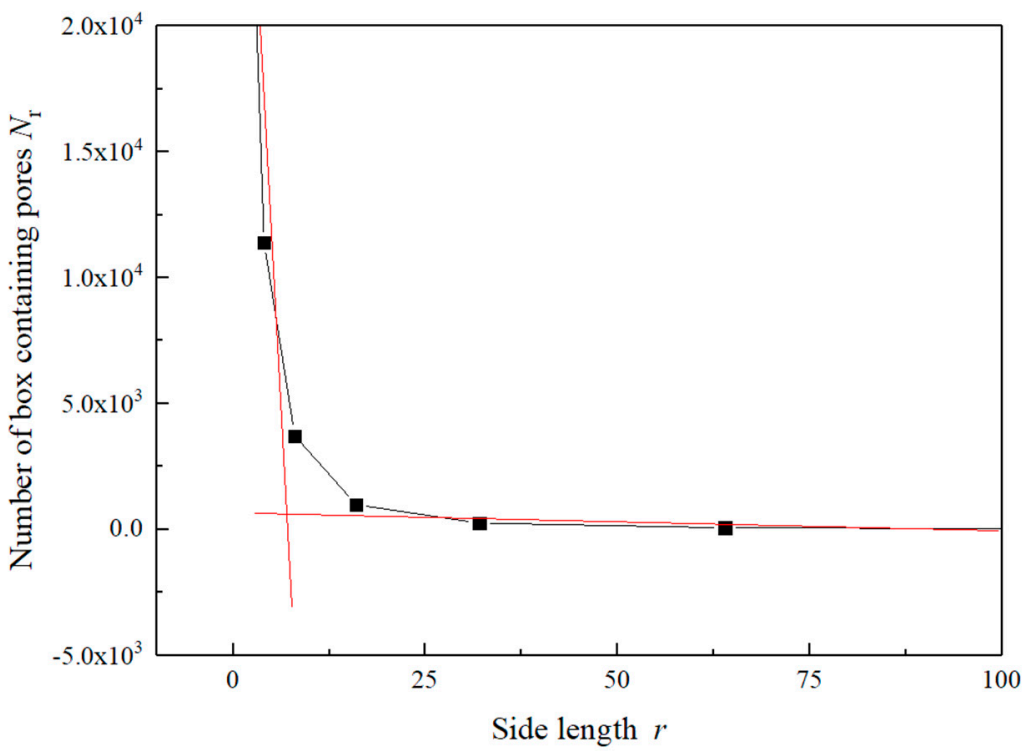

Figure 12. Distribution of the side length against the number of boxes containing pores.

Figure 12 indicates that when the edge length of the box exceeds 4.1 (i.e., $r>4.1$ ), the total number of boxes is small, the edge length of the box is almost constant. At this stage, boxes containing porous parts and boxes containing entities overlap with each other. In fact, Figure 13a shows that there are both holes and entities in each box. On the other hand, when the edge length of the box is lower than 4.1 (i.e., $r<4.1$ ), the total number of boxes dramatically increases, which indicates that boxes containing entities and boxes containing holes are separated. In other words, either a box is an entity or contains an entity with only a small part of the pore, or a box is entirely porous or contains only a small part of the entity at the same time. This is more pronounced when the edge length of the box continues to decrease. At this stage, Figure $13 \mathrm{~b}$ shows that most of the boxes are located inside the target (entity or pore). Therefore, the intersection point fitted by the straight line from the initial stage and the descending stage can be regarded as the critical point for separating the entity and the pore. The actual size corresponding to the edge length of a single box at the critical point can be approximated as the maximum diameter of the pore. Thus, for the box coverage data shown in Table 1, when the box size is $r=4.1$, the actual size corresponds to the maximum pore diameter at that time. The same method can be applied to calculate the maximum pore diameter for the sealing interface of the porous media under other conditions.

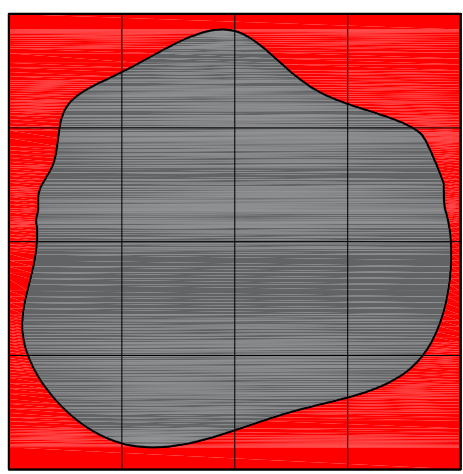

(a)

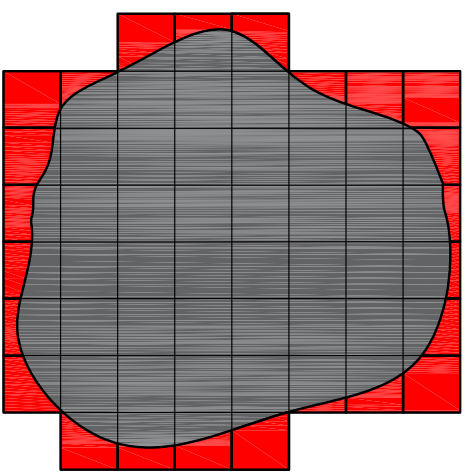

(b)

Figure 13. The schematic sketch of the pore covering at different side lengths: (a) $r>4.1 ;$ (b) $r<4.1$.

Figure 14 shows the distribution of the maximum pore diameter of the porous media for the sealing interface at different contact pressures. It is found that the maximum pore 
diameter decreases gradually as the contact pressure increases. Moreover, at constant extrusion of external loads, more rough peaks are flattened and filled with the pore part, which further reduces the maximum pore diameter.

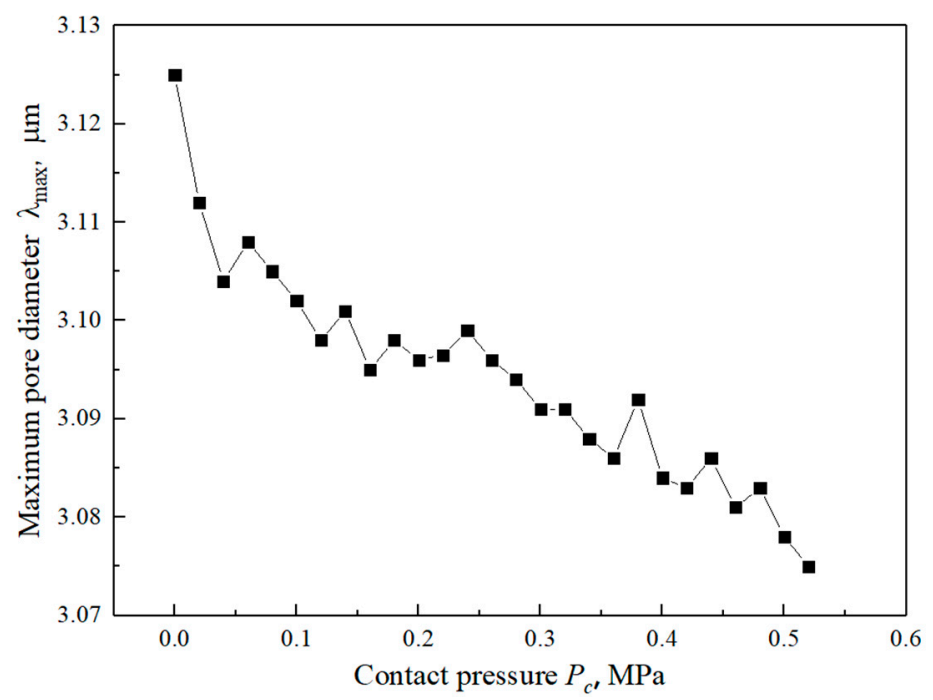

Figure 14. Distribution of the contact pressure against the maximum pore.

\subsection{Feasibility Analysis of the Model-Based Leakage Rate Calculation for Contact Mechanical Seals}

In order to illustrate the feasibility of using the proposed model to calculate the leakage rate of contact mechanical seals, results from the conventional model for the parallel plane slot flow [5] and the theoretical percolation model [17] are analyzed. It should be indicated that the following parameters are utilized in the present calculation: elastic modulus $E=2500 \mathrm{MPa}$, Poisson's ratio $v=0.3$, the surface roughness $R_{\mathrm{a}}=0.25 \mu \mathrm{m}$, pressure difference inside and outside the sealing interface $\Delta P=0.2 \mathrm{MPa}$, and the sealing medium is water. The dynamic viscosity of the fluid at $20{ }^{\circ} \mathrm{C}$ is $\mu=1.005 \times 10^{-3} \mathrm{~Pa} \cdot \mathrm{s}$. According to Equation (12) and the above-mentioned calculation methods of the porous media interface construction and microstructural parameters of mechanical seals, the leakage rate of the interface can be calculated when the contact pressure of the end surface varies from 0.05 to $0.5 \mathrm{MPa}$.

Figure 15 shows the variation of the leakage rate in three models. It is found that the calculated leakage rate from three models decreases as the contact pressure increases, which is consistent with the actual situation. Moreover, the calculated results of the three models at the same contact pressure are in the same order of magnitude. In other words, the calculated leakage rate by the parallel plane slot flow model is the largest, while the calculated leakage rate by the percolation theory model is the lowest. Furthermore, it is found that the results obtained from the proposed fractal model of the porous media are located between those obtained from the two foregoing methods. The difference is that the obtained results can be interpreted as the following: The parallel plane slot flow model simplifies the rough and uneven seal face into an ideal smooth plane and ignores the influence of the end shape on the leakage rate. Therefore, the corresponding calculated leakage rate is higher than the actual value. The calculation of the leakage rate based on the theoretical percolation model is mainly focused on the leakage at a single percolation point, while there may be multiple percolation points and different bifurcations that may form in the actual leakage passage. Therefore, the corresponding calculated leakage rate is smaller than the actual value. Based on data for the real mechanical seal interface, the geometric model of the porous media is constructed in accordance with the characteristics of the porous media. With the help of fractal and porous media transport theory, the present model can objectively reflect the flow of the interfacial fluid from a microscopic perspective. Therefore, it has better physical significance and rationality. 


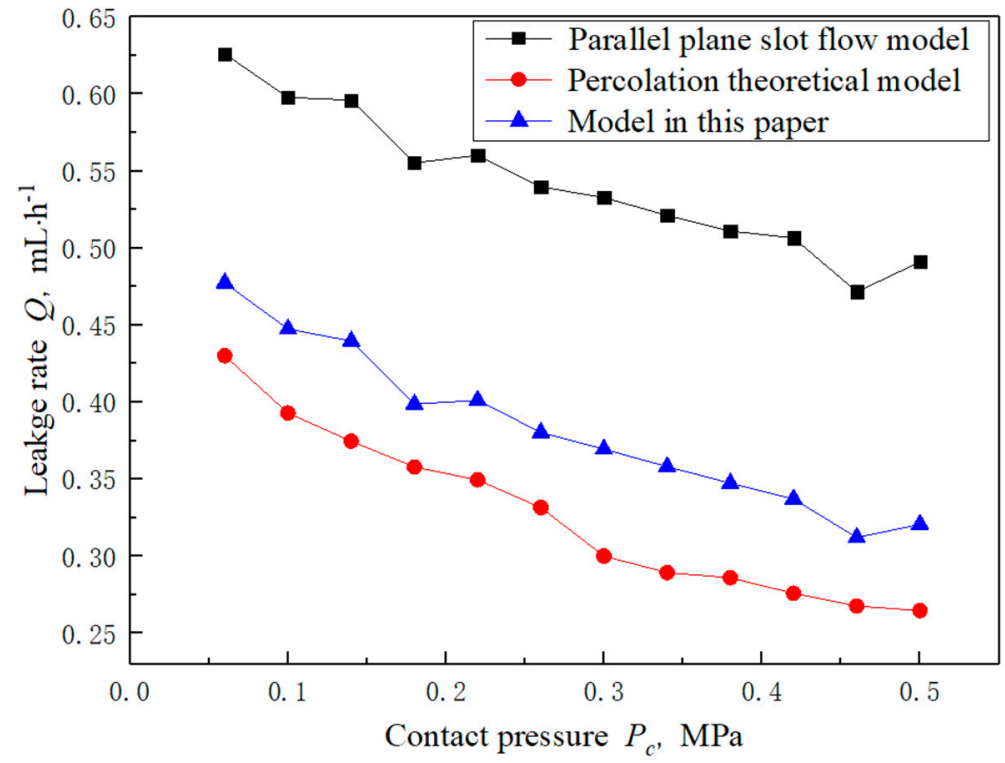

Figure 15. Comparison of leakage rates calculated using different models.

Obviously, it is best if the model results can be verified by the experimental results. At present, the test is being carried out, mainly through the static test method to obtain the leakage rate under different loads to verify the correctness of the theory. In the next theoretical research work, it is necessary to further consider the fluid bearing and different contact characteristics, as well as the influence of friction and wear on the morphology and representative area of the seal ring in the relative operation process.

\section{Conclusions}

Based on the fractal theory of the porous media, a theoretical model for calculating the leakage rate of the seal face is established in the present study. The proposed method is based on the modified Hagen-Poiseuille equation by using the real contact mechanical seal interface. The obtained results have certain reference significance for calculation and prediction of the leakage rate and in-depth study of the leakage mechanism.

The main achievements and conclusions of the present study are as the following:

- According to the principle of reverse engineering, the morphological data of the real seal face are collected, and the geometric model of the porous media is constructed based on the contact model of dynamic and static rings and the analysis of the elasticplastic deformation law.

- The method is proposed for calculating the microstructural parameters of the porous media model with the sealed interface, including the porosity, fractal dimension of the pore area and the maximum pore diameter. Especially, a novel method is proposed for calculating the maximum pore diameter based on the box-counting method. Furthermore, the variation of parameters at different contact pressures is analyzed. The results show that the porosity and maximum pore diameter decrease gradually as the contact pressure increases, while the fractal dimension of the pore area increases gradually.

- The leakage rate predicted by the proposed model and two conventional models are of the same order, which validates the feasibility of the proposed model.

Author Contributions: Data curation, X.N. and C.M.; formal analysis, Y.Z.; investigation, C.M.; methodology, C.M.; resources, Y.Z.; software, Q.Y.; supervision, J.S.; validation, C.M.; visualization, X.N. and J.S.; writing—original draft, C.M.; writing—review and editing, X.N. All authors have read and agreed to the published version of the manuscript. 
Funding: This work was supported by the National Key R\&D Program of China (2018YFB2000800); Postgraduate Research \& Practice Innovation Program of Jiangsu Province (KYCX18_0970); Key R\&D Program of Jiangsu Province (BE2017026); Natural Science Foundation of China (51805269); and Youth Science and Technology Innovation Foundation of Nanjing Forestry University (CX2017007).

Data Availability Statement: Data sharing is not applicable to this article.

Conflicts of Interest: The authors declare no conflict of interest.

\section{Symbol Description}

$\begin{array}{ll}M(L) & \text { Measurement of fractal objects } \\ L & \text { Measurement scale } \\ D_{f} & \text { Fractal dimension } \\ N & \text { Distribution number of pores } \\ \lambda, \lambda_{\max } \text { and } \lambda_{\min } & \text { Normal, maximum and the minimum pore } \\ \Delta P & \text { diameters, } \mathrm{m} \\ \mu & \text { Pressure of the sealing medium, Pa } \\ L_{0} & \text { Hydrodynamic viscosity, Pa.S } \\ \tau_{a v} & \text { Representative length of the channel, } \mathrm{m} \\ Q & \text { Average tortuosity } \\ \phi & \text { Quantity of flow, } \mathrm{m}^{3} \cdot \mathrm{s}^{-1}\end{array}$

\section{References}

1. Mayer, E. Mechanical Seals, 6th ed.; Chemical Industry Press: Shanghai, China, 1981.

2. Lebeck, A.O. How much do we know about mechanical seals. Seal. Technol. 2006, 9, 11-12. [CrossRef]

3. Green, I. On the kinematics and kinetics of mechanical seals, rotors, and wobbling bodies. Mech. Mach. Theory 2008, 43, 909-917. [CrossRef]

4. Lu, J. Study on Flow Characteristics of Leakage Fluid between Contact Mechanical Seals; Nanjing Forestry University: Jiangsu, China, 2017.

5. Fan, Y.E.; Gu, F.; Ball, A. A review of the condition monitoring of mechanical seals. In Proceedings of the ASME, Biennial Conference on Engineering Systems Design and Analysis, Manchester, UK, 19-22 July 2004; pp. 179-184.

6. Gu, Y. Fluid Dynamic Seal; Petroleum University Press: Chengdu, China, 1990.

7. Heinze, E. Besonderer berbcksichti-dung ihrer verwendung im kältema schinenbau. Kältetechnik 1949, 1, 26-32. (in German).

8. Lebeck, A.O. Hydrodynamic lubrication in wavy contacting face seals: A two dimensional model. J. Tribol. 1981, 103, 578. [CrossRef]

9. Lebeck, A.O. Contacting mechanical seal design using a simple hydrostatic model. Tribol. Int. 1988, 21, 2-14. [CrossRef]

10. Elhanafi, S.; Farhang, K. Leakage prediction in mechanical seals under hydrostatic operating condition. In Proceedings of the ASME/STLE International Joint Tribology Conference, San Diego, CA, USA, 22-24 October 2007; pp. 505-507.

11. Green, I. A transient dynamic analysis of mechanical seals including asperity contact and face deformation. ASLE Trans. 2002, 45, 284-293. [CrossRef]

12. Sun, J.; Wei, L.; Feng, X.; Gu, B. Leakage prediction method for contacting mechanical seals with parallel faces. Chin. J. Mech. Eng. 2010, 23, 7-15. [CrossRef]

13. Pérez-Ràfols, F.; Larsson, R.; Almqvist, A. Modelling of leakage on metal-to-metal seals. Tribol. Int. 2016, 94, 421-427. [CrossRef]

14. Li, X.; Yang, Z.; Xu, J.; Chen, R.; Yang, H. The fractal leakage model of contact mechanical seals considering wear and thermal deformation. J. Braz. Soc. Mech. Sci. Eng. 2019, 41, 1-12. [CrossRef]

15. Wei, L.; Chang, X.Z.; Zhang, P. Research progress on end leakage model of contact mechanical seals. Fluid Mach. 2012, 40, 36-40.

16. Bao, C.; Meng, X.; Li, J.; Peng, X. Leakage rate of liquid lubrication mechanical seals based on percolation principle. Fluid Mach. 2014, 24-28.

17. Bottiglione, F.; Carbone, G.; Mantriota, G. Fluid leakage in seals: An approach based on percolation theory. Tribol. Int. 2009, 42, 731-737. [CrossRef]

18. Yu, Q. Study on the Performance of Contact Mechanical Seals; Nanjing Forestry University: Nanjing, China, 2014.

19. Zhou, M. Percolation Mechanism and Channel Simulation of Contact Mechanical Seal Interface; Nanjing Forestry University: Nanjing, China, 2015.

20. Sun, J.; Ma, C.; Lu, J.; Yu, Q. A leakage channel model for sealing interface of mechanical face seals based on percolation theory. Tribol. Int. 2018, 118, 108-119.

21. Ji, Z.B.; Sun, J.J.; Ma, C.B.; Yu, Q.; Lu, J. Static leakage prediction method of mechanical seal interface based on percolation theory. CIESC J. 2017, 6, 734-742.

22. Patel, H.; Hariharan, H.; Bailey, G.; Jung, G. Advanced Computer Modelling for Metal-to-Metal Seal in API Flanges. In Proceedings of the Society of Petroleum Engineers Annual Technical Conference and Exhibition, Dallas, Texas, 24-26 September 2018. 
23. Zhang, Q.; Chen, X.; Huang, Y.; Chen, Y. Fractal modeling of fluidic leakage through metal sealing surfaces. AIP Adv. 2018, 8, 045310. [CrossRef]

24. Bell, D.J. Hydrodynamics of Porous Media; Construction Industry Press: Beijing, China, 1983.

25. Mandelbrot, B.B.; Wheeler, J.A. The fractal geometry of nature. Am. J. Phys. 1983, 51, 286. [CrossRef]

26. Sahimi, M. Flow and Transport in Porous Media and Fractured Rock: From Classical Methods to Modern Approaches; VCH: Vancouver, BC, Canada, 2011.

27. Majumdar, A.; Bhushan, B. Role of fractal geometry in roughness characterization and contact mechanics of surfaces. J. Tribol. 1990, 112, 205-216. [CrossRef]

28. Yu, B.; Li, J. Erratum: Some fractal characters of porous media. Fractals Complex Geom. Patterns Scaling Nat. Soc. 2002, 10, 135. [CrossRef]

29. Yu, B.; Lee, L.J.; Cao, H. A fractal in-plane permeability model for fabrics. Polym. Compos. 2002, 23, 201-221. [CrossRef]

30. Yu, B.; Cheng, P. Fractal models for the effective thermal conductivity of bi-dispersed porous media. J. Thermophys. Heat Transf. 2002, 16, 22-29. [CrossRef]

31. Yu, B.M.; Xu, P.; Zou, M.Q.; Cai, J.C.; Zheng, Q. Transport Physics of Fractal Porous Media; Science Press: Beijing, China, 2014.

32. Katz, A.J.; Thompson, A.H. Fractal sandstone pores: Implications for conductivity and pore formation. Phys. Rev. Lett. 1985, 54, 1325-1328. [CrossRef] [PubMed]

33. Liao, C.; Chen, H.; Lu, H.; Dong, R.; Sun, H.; Chang, X. A leakage model for a seal-on-seal structure based on porous media method. Int. J. Press. Vessel. Piping 2020, 188, 104227. [CrossRef]

34. Wang, S.; Yao, X.F.; Yang, H.; Yuan, L.; Dong, Y.F. A new leakage measurement method for damaged seal material. Meas. Sci. Technol. 2018, 29, 075203. [CrossRef] 